\title{
Microanatomical Features of the Uterus of Albino Wistar Rat (Rattus norvegicus) in Oestrous
}

\author{
Archana Kantepudi, Ranjith Kumar Sundari" and Rajendranath Nalla \\ Department of Veterinary Anatomy, College of Veterinary Science, Rajendranagar, Hyderabad, INDIA \\ *Corresponding author: RK Sundari; E-mail:varma.dr@gmail.com
}

Received: 06 May, 2021

Revised: 07 June, 2021

Accepted: 16 June, 2021

\begin{abstract}
This study describes histology of uterus in the Albino Wistar rat induced with oestrous. Twenty adult female Wistar rats were used in the study. Ten rats were induced with estrous using Diethylstilbestrol $1 \mathrm{mg} / \mathrm{kg}$ b.wt subcutaneously for three consecutive days. Uteri were obtained from both groups after three days upon induction of diestrous stage. Endometrial histology in both the groups displayed glandular mucosa comprising of lamina epithelialis and lamina propria mucosae. The epithelium was stratified cuboidal with numerous neutrophils and lymphocytes in the lamina propria mucosa. The cellularity was more in the estrous induced group. Lamina muscularis was absent in the tract of both groups. The uterine glands occurred in variable numbers and sizes in the propria-submucosae of the estrous induced group of rat uterii. These glands were lined by simple cuboidal epithelium. The myometrium consisted of inner circular and outer longitudinal layer wherein the smooth circular layer was comparatively thicker than the longitudinal layer in both groups. Stratum vasculare containing prominent blood vessels was well appreciated between the two muscular layers. The perimetrium comprised of simple squamous epithelium.
\end{abstract}

\section{HIGHLIGHTS}

(0 The cellularity was more in the lamina propria mucosae of the estrous induced group.

0 Lamina propria contained numerous blood vessels and endometrial glands during estrus.

(0 Vasculature was more appreciated in the oestrous induced group.

Keywords: Oestrous, Uterus, Albino rat

The rat has been elected as the main animal model in several studies involving reproduction. Female rodents are poliestric, present spontaneous ovulation and show regular and successive estrous cycles that may vary with age and species. These cycles are also influenced by light, seasons of the year and life circumstances. On the other hand, estrous cycles occur without seasonal influence in rats submitted to environmental control under laboratory conditions (Lohmiller and Swing, 2006). Akinloye and Oke (2015) had characterized the uterus of the African giant rat as a duplex uterus having two separate uterine horns and two cervices. Estrous cycles are characterized by morphological changes in ovaries, the uterus and the vagina (Goldman et al., 2007) which occur during different phases called proestrus, estrus, metestrus and diestrus. This study is aimed to provide a baseline for understanding the normal appearance of the uterus in diestrus which will allow one to differentiate otherwise normal reproductive tissues with delayed development (i.e., sexual immaturity) from reproductive tissues with direct or specific test substance-related lesions.

Twenty adult female rats of Wistar origin aged 80-100 days and weighing approximately $200 \mathrm{~g}$ were used in this study. The animals were housed in temperature-controlled rooms,

How to cite this article: Kantepudi, A., Sundari, R.K. and Nalla, R. (2021). Microanatomical Features of the Uterus of Albino Wistar Rat (Rattus norvegicus) in Oestrous. J. Anim. Res., 11(04): 761-764.

Source of Support: None; Conflict of Interest: None क्) 
with a light regime of 12-h light and 12-h dark. Food and water were provided ad libitum. Oestrous was induced in ten rats using Diethyl stilbesterol subcutaneously@1mg/ $\mathrm{kg}$ body weight for three days subsequently. They were sacrificed on the third day following standard CPCSEA protocol and uterii were dissected from the control group (C) and from the group induced with estrous (T). The organs were washed with water to remove the blood stains and debris. Thereafter, uterine samples were collected and processed for light microscopy. Tissues were formalinfixed, trimmed, paraffin-embedded, sectioned at 5- $\mu \mathrm{m}$ thickness, and examined microscopically. The Ethics Committee of the University approved this study.

The Albino Wistar rat's uterus was duplex, i.e. it comprised of two uterine horns which joined together and opened into the vagina via two separate cervical canals which was in agreement with the observations of Akinloye and Oke (2015) in African giant rat. Two uterine horns or tubes joined caudally in a Y fashion. Cervix can be arbitrarily divided into cranial and caudal parts. The cranial part of cervix became wide and swollen at its junction with the horns (Fig. 1). Internally it contained two lumina separated by a midline septum (Fig. 2).

The narrow and slightly tapered caudal part of the cervix projects into the cranial portion of the vagina. Uterus was engorged in appearance in the group induced with estrus (Fig. 1).

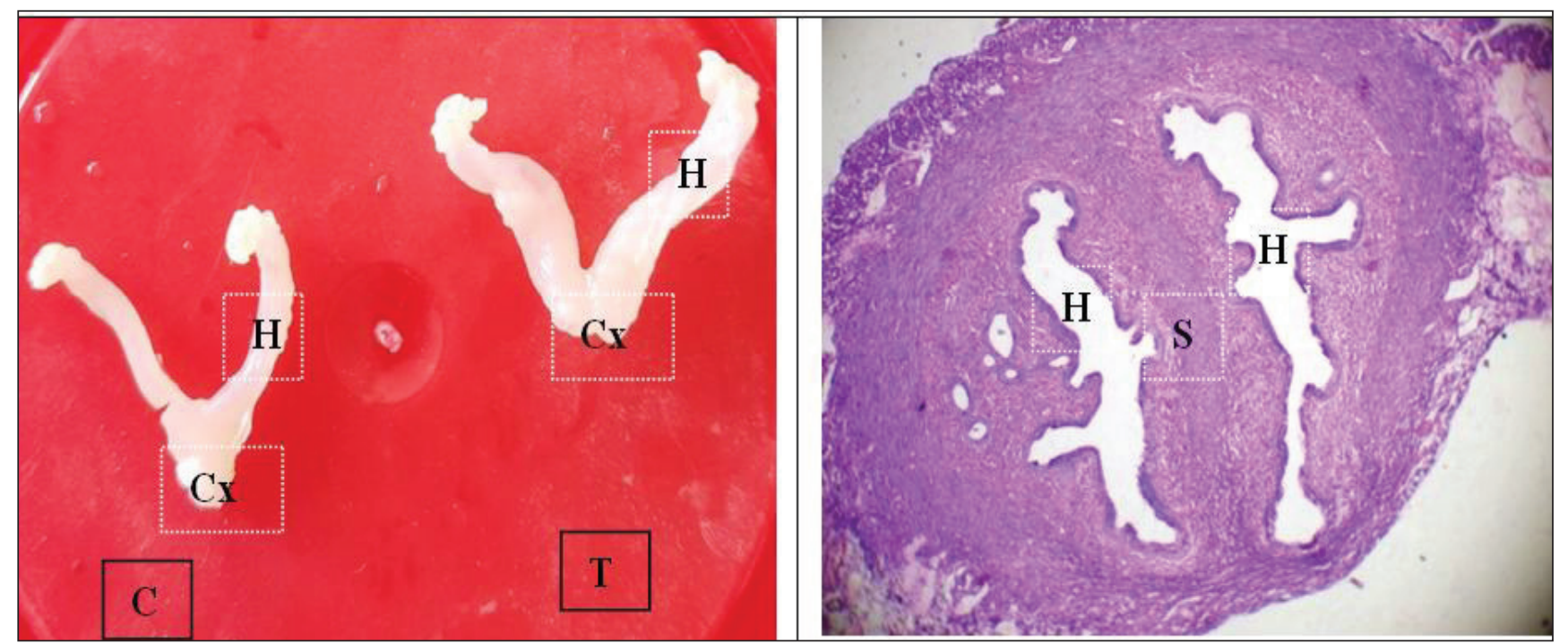

Fig. 1: Gross photograph of the normal uterus (C) and uterus induced with estrus $(\mathrm{T})$ Uterine horn $(\mathrm{H})$ Cervix $(\mathrm{Cx})$
The inner mucosa, or endometrium, consisted of a surface stratified cuboidal epithelium in both the groups while the stratification was more obvious in the folds of the uterine wall (Fig. 3). Lamina propria contained numerous blood vessels and endometrial glands during estrus which were akin to observations of Geoffrey O. Wood et al. (2007) who observed that greatest stromal proliferation during estrus in mice.

In contrast to the looser and more abundant, cellular and vascular endometrial stroma of the uterine horns, the endocervical stroma of the cervix was composed primarily of thick, interlacing bundles of compactly arranged collagenous fibers (Fig. 4). Variable numbers of lymphocytes and other leucocytes were present within the superficial lamina propria of the endometrium in both the groups which concur with the findings of (Geoffrey O.Wood et al., 2007). The uterine gland or endometrial gland was a simple tubular gland formed by invagination of the uterine endometrium (Mosavat et al., 2019). Uterine glands were fewer in the control group but were plenty in the estrous induced group (Fig. 5). Lamina muscularis was absent. The middle muscular layer or myometrium was composed of an inner circular and outer longitudinal smooth muscle layer. The circular layer was comparatively thicker than the longitudinal layer in both the groups which is in accordance with the observations of Catherine et al. (2016) (Fig. 6). Stratum vasculare comprising of

Fig. 2: Photomicrograph of the cervical region showing the two uterine horns $(\mathrm{H})$ seperated by a septum (S). H\&E 10X 


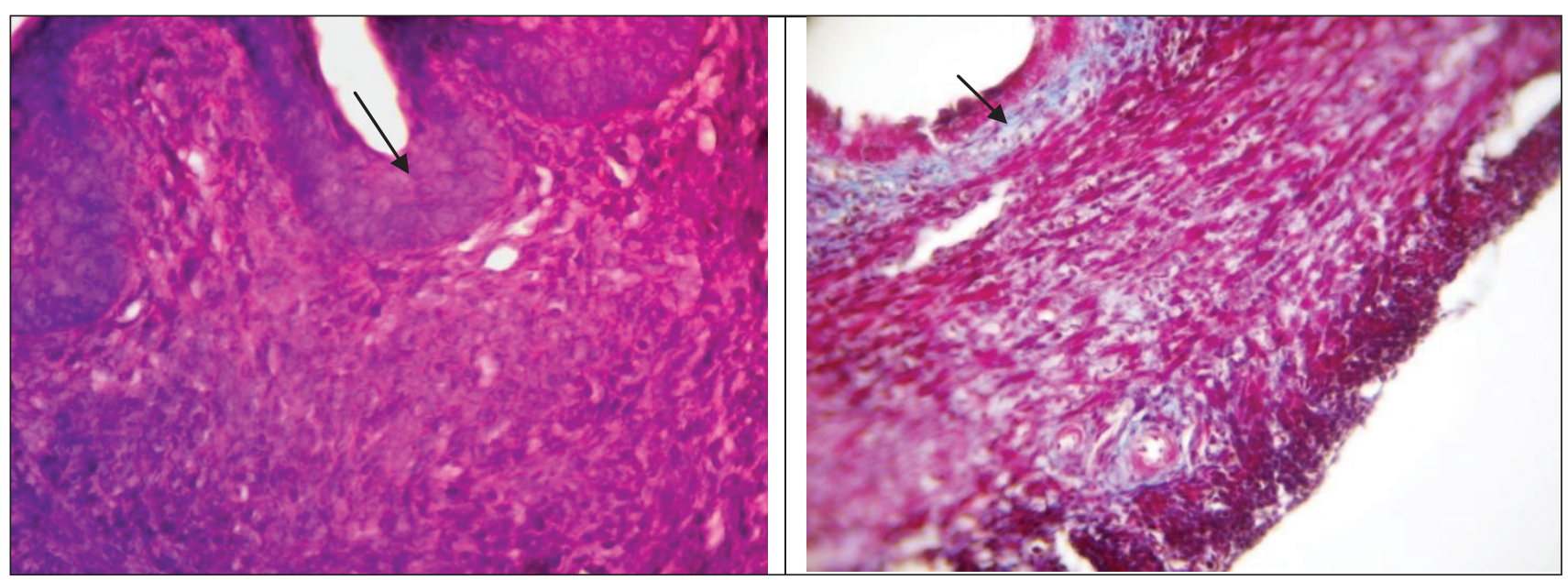

Fig. 3: Photomicrograph of the uterine horn showing the stratified Fig. 4: Photomicrograph of cervix showing collagen fibres cuboidal epithelium in control group. H\&E 40X (arrow) in the lamina propria

\section{MASSON'S TRICHROME 10X}

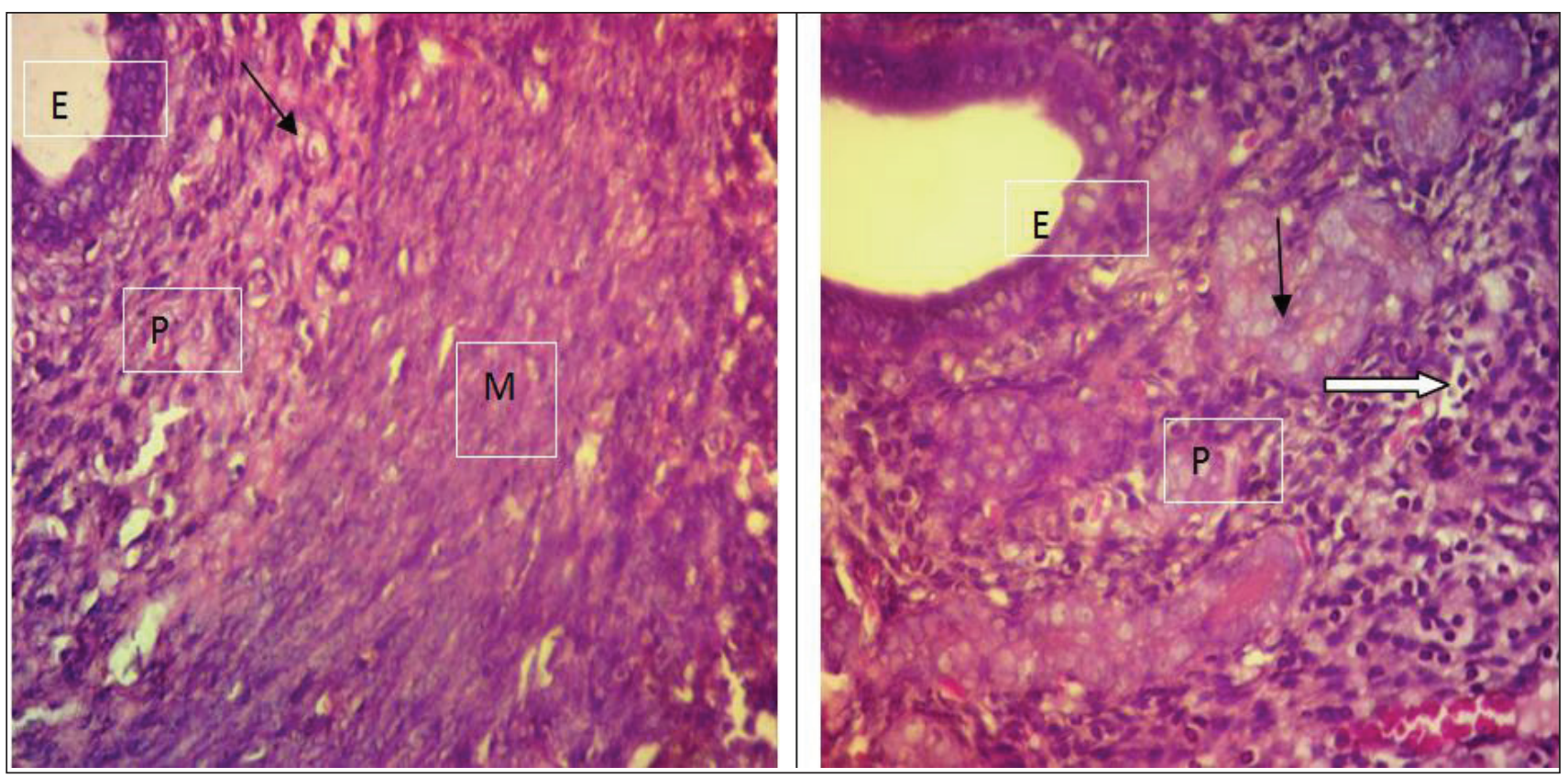

Fig. 5: Photomicrograph of uterine horn showing few endometrial glands in control group and numerous tubular glands (black arrow), lymphocytes (white arrow) in the oestrous induced group. E- Epithelium, P- Propria submucosa, M- Muscular layer H\&E $40 \mathrm{X}$

numerous blood vessels was present between the two muscular layers. Vasculature was more appreciated in the oestrous induced group (Fig. 7). The medial part of each circular layer merged to blend with a mid-sagittal sheet of longitudinally arranged smooth muscle fibers that occupied a central position in the midline septum (Fig. 2) in the cranial part of cervix. The myometrium was covered by the perimetrium, a thin connective tissue layer overlain by a simple serosa. 


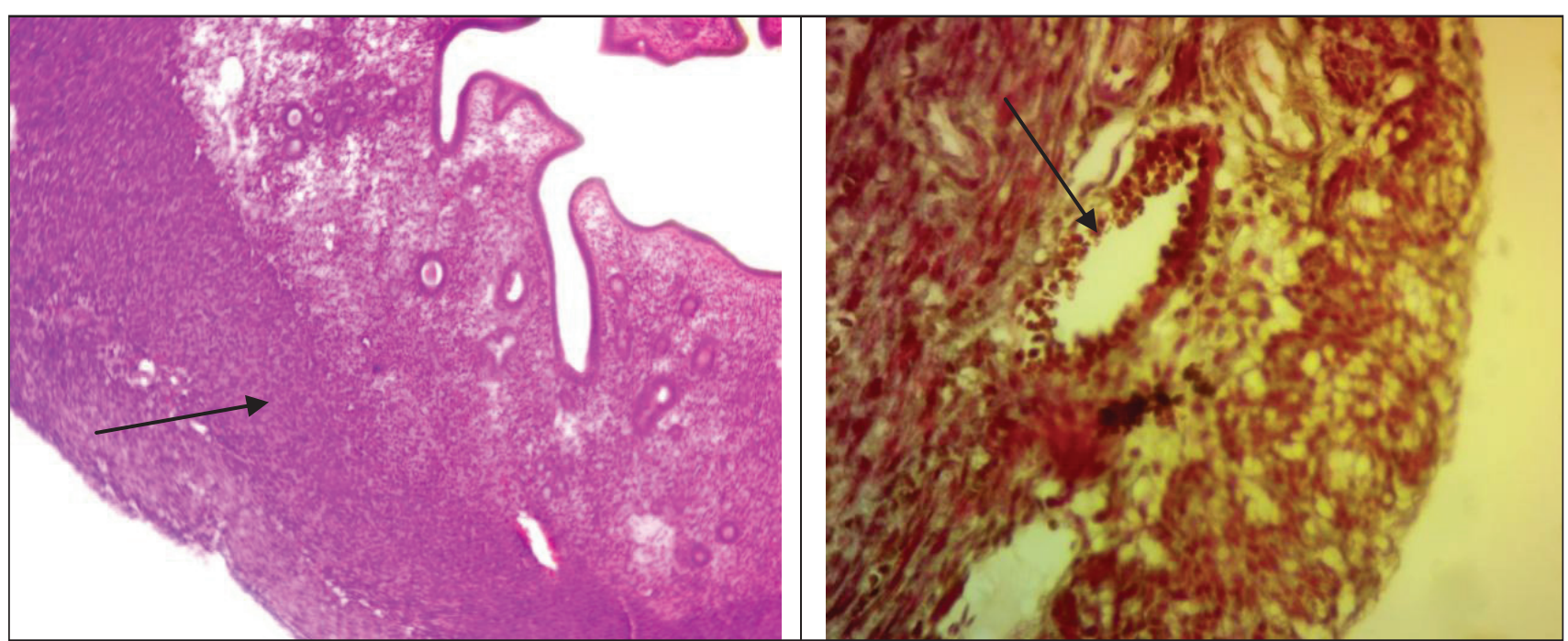

Fig. 6: Photomicrograph showing thicker circular muscle layer (arrow) of the myometrium in estrous induced group H\&E 40X

Fig. 7: Photomicrograph showing stratum vasculare in estrous induced group. VANGEISON'S STAIN 40X

\section{CONCLUSION}

In conclusion, the uterine histological structure varied during oestrous cycle. Uterus was engorged in appearance in the group induced with estrus. Uterine glands number increased in oestrous. Lamina propria contained numerous blood vessels and endometrial glands during estrus. Vasculature was more appreciated in the oestrous induced group. This study complements previous findings obtained in the uterus during oestrous cycle the rat. These findings also provide baseline information for future molecular research in the uterus of the Albino Wistar rat.

\section{REFERENCES}

Akinloye, A.K. and Oke, B.O. 2015. Histology and ultrastructure of the uterus of African giant rat (Cricetomys Gambianus, Waterhouse) during oestrous cycle. Folia Morphol., 74(3): 311-317.

Catherine A. Picut and Amera K. Remick. 2016. Female Reproductive System. Atlas of Histology of the Juvenile Rat. 203-226.

Geoffrey O. Wood, Jimmie E Fata, Katrina LM Watson and Khokha, R. 2007. Circulating hormones and estrous stage predict cellular and stromal remodeling in murine uterus. Reproduction, 133(5): 1035-1044.

Goldman, J.M., Murr, A.S. and Cooper, R.L. 2007. The rodent estrous cycle: characterization of vaginal cytology and its utility in toxicological studies. Birth Defects Res. B Dev. Reprod. Toxicol., 80: 84-97.

Lohmiller, J.J. and Swing, S.P. 2006. Reproduction and breeding. In: Suckow MA, Weisbroth SH, Franklin CL. The Laboratory Rat. $2^{\text {nd }}$ ed. New York, NY: Academic Press, pp. 147-162.

Mosavat, M., Mohamed, M., Ooi, F.K., Mirsanjari, M., Zin, A.A.M. and Romli, A.C. 2019. Histological changes of female reproductive organs subjected to different jumping exercise intensities and honey supplementation in rats. Peer $J ., 7$ : e7646. 